\title{
Recent Trends in Fusion Gyrotron Development at KIT
}

\author{
G. Gantenbein*, K. Avramidis, J. Franck, S. Illy, Z. C. Ioannidis, J. Jin, J. Jelonnek, P. Kalaria, \\ I. Gr. Pagonakis, S. Ruess, T. Rzesnicki, M. Thumm, C. Wu
}

Karlsruhe Institute of Technology (KIT), IHM, Kaiserstr. 12, 76131 Karlsruhe, Germany

\begin{abstract}
ECRH\&CD is one of the favorite heating system for magnetically confined nuclear fusion plasmas. KIT is strongly involved in the development of high power gyrotrons for use in ECRH systems for nuclear fusion. KIT is upgrading the sub-components of the existing $2 \mathrm{MW}, 170 \mathrm{GHz}$ coaxial-cavity short-pulse gyrotron to support long-pulse operation up to $1 \mathrm{~s}$, all components will be equipped with a specific active cooling system. Two important developments for future high power, highly efficient gyrotrons will be discussed: design of gyrotrons with high operating frequency $(\sim 240 \mathrm{GHz})$ and efficiency enhancement by using advanced collector designs with multi-staged voltage depression.
\end{abstract}

\section{Introduction}

This contribution reports on activities in the field of research and development of RF sources (gyrotrons) and related components for electron cyclotron resonance heating and current drive (ECRH\&CD) of magnetically confined nuclear fusion plasmas at KIT. In particular activities towards a $170 \mathrm{GHz}, 2 \mathrm{MW}$ gyrotron for a later stage of ITER and developments towards a future DEMO reactor will be addressed.

KIT is pushing forward the experimental verification of the multi-megawatt coaxial-cavity technology, by upgrading the $170 \mathrm{GHz} 2 \mathrm{MW}$ coaxial-cavity short-pulse prototype to longer pulses (up to $1 \mathrm{~s}$ ). In order to increase the pulse length of the $2 \mathrm{MW}$ coaxial cavity gyrotron all major subcomponents have been equipped with active cooling systems. An improved design of a new Magnetron Injection Gun (MIG) which respects fundamental gun design criteria for long-pulse operation and minimises beam instabilities has been performed and ordered. An inverse MIG (IMIG) has been designed and is currently under manufacturing. Several advantages of this type of gun compared to a conventional MIG will be verified experimentally.

In the frame of EUROfusion the investigation of advanced $240 \mathrm{GHz}$ gyrotrons for future DEMO is ongoing, focusing on two options: conventional-cavity gyrotron and coaxial-cavity gyrotron. Conventionalcavity gyrotrons offer a robust design with, at present, 1 MW RF output power. Our investigations on limits of that design show that $1.5 \mathrm{MW}$ could be achieved with this technology. The coaxial cavity technology is more challenging, but offering higher output power. A focus area in the development is the investigation of the impact of tolerances and misalignment of the electron beam and coaxial insert on stable operation of the design mode.

High efficiency operation of gyrotrons is a key issue for future application in ECRH systems of fusion reactor relevant devices. To operate such a system economically an overall efficiency of the gyrotron significantly above $50 \%$ is required. KIT is focussing on the developments of advanced collectors which recover efficiently the nonspent energy of the electron beam. Several principles of multistage depressed collector systems (MSDC) have been studied intensively.

\section{MW Coaxial Cavity Gyrotron Development}

In order to increase the gyrotron pulse length of the existing $2 \mathrm{MW}$ coaxial-cavity gyrotron [1], all subcomponents such as beam tunnel, cavity, launcher, mirrors, synthetic diamond window, and the collector will be equipped with an active cooling system. In order to take benefit of the modularity of the gyrotron independent cooling systems have been designed. This allows monitoring of the internal losses of the individual components and an energy balance of the tube during long-pulse operation.

The design of the existing conventional magnetron injection gun (MIG) has been improved in order to support the design criteria for long-pulse operation [2]. The suppression of the electron beam halo caused by magnetically trapped electrons in the region between the cathode and the cavity has been optimized by modification of the anode and cathode contour. The

\footnotetext{
Corresponding author: gerd.gantenbein@kit.edu
} 


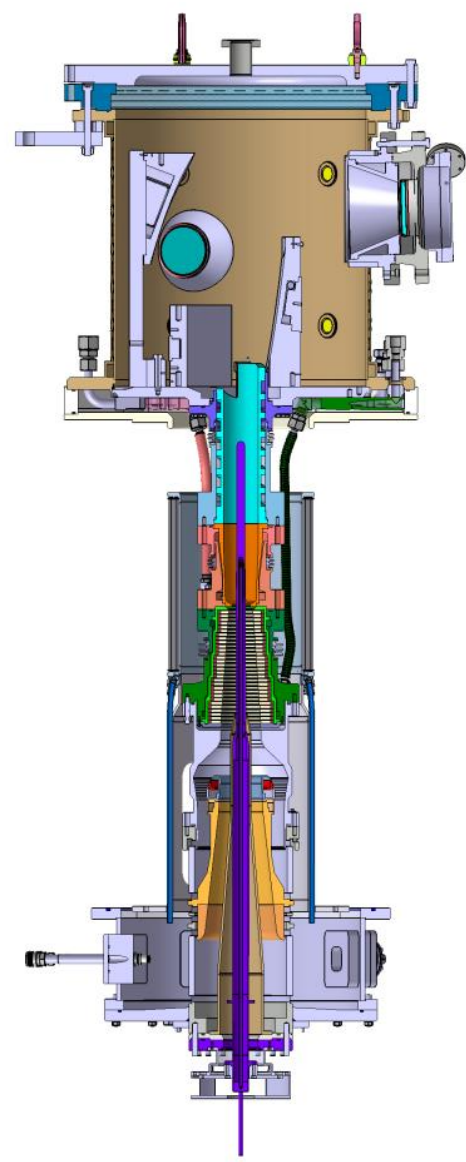

a)

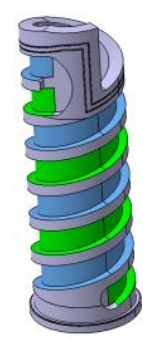

b)

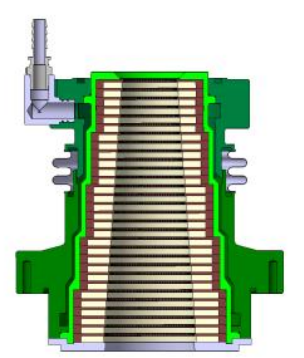

d)

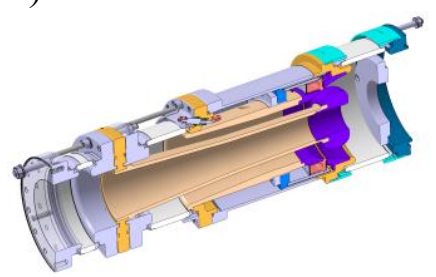

f)

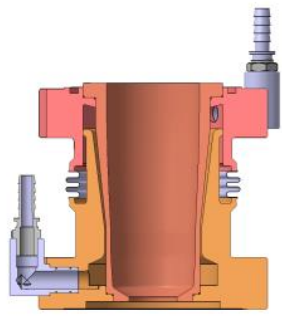

c)

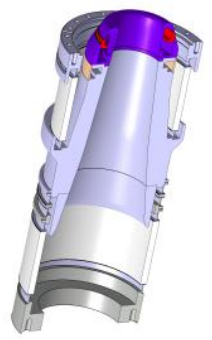

e)

Fig. 1:Schematic view of coaxial cavity gyrotron (a) with long-pulse components, launcher (b), cavity (c), beam tunnel (d), MIG (e) and IMIG (f).

generation of secondary electrons in non-emitter regions from bombardment with trapped particles has been minimised. Additionally, an advanced type of emitter ring [3] is used in order to minimize the sensitivity of the manufacturing and alignement tolerances of the emitter and its neighbouring parts on the gyrotron performance In parallel, an innovative Inverse Magnetron Injection Gun (IMIG) has been designed at KIT for the modular $2 \mathrm{MW}, 170 \mathrm{GHz}$ coaxial-cavity pre-prototype gyrotron [4]. The design allows the implementation of a significant larger emitter ring compared to the conventional MIG, considering the same radial tube dimensions. Due to the direct cooling of the thermally loaded components close to the emitter a better temperature profile for those components and a smaller thermal expansion of the beam forming components is achieved which reduces the possibility for unwanted emission.

A traditional beam tunnel with stacked corrugated copper and damping ceramic rings and active cooling has been designed. The development and manufacturing of a full metallic beam tunnel is also in process.

$\mathrm{RF}$ power generation from electron-wave interaction takes place in the cavity and is necessarily associated with ohmic surface losses. In this case the power loss at the surface of the cavity will be $50 \mathrm{~kW}$ with a maximum power density $\sim 2 \mathrm{~kW} / \mathrm{cm}^{2}$ at the centre of the cavity. The approach of the active cooling system of the cavity is shown in Fig. 1. The outer water jacket is movable along the axis in order to respect the different temperature and thermal expansion coefficient of the components.

The launcher and the mirror system are responsible for the conversion of the main $\mathrm{TE}_{34,19}$ cavity mode into a linearly polarized fundamental Gaussian beam mode. In high power operation the loading of the launcher surface, especially at the top part of the launcher, is very high. A

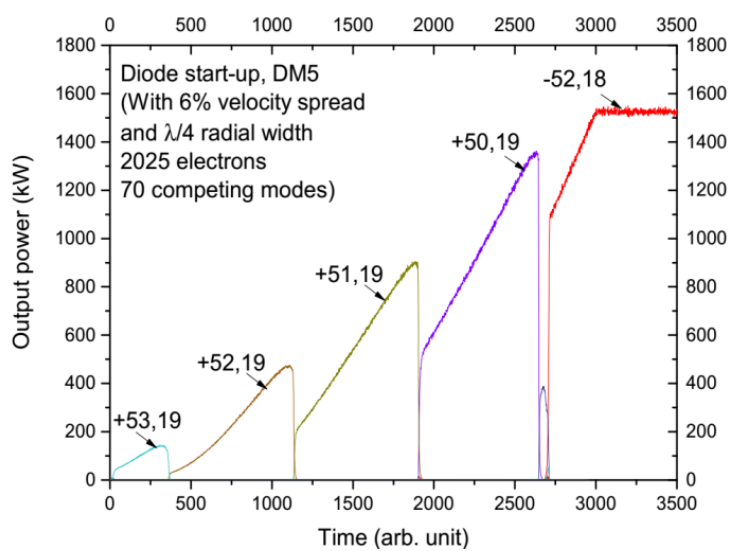

Fig. 2: Multi-mode, self-consistent, diode start-up analysis for $\mathrm{TE}_{52,18}$ mode. 
helical cooling structure has been designed in order to guarantee stable operation.

\section{$3240 \mathrm{GHz}$ Gyrotron Development for DEMO}

Based on the (EU-) DEMO baseline of 2012 design studies on gyrotrons and key components have been performed in order to identify and overcome possible design and simulation obstacles and limitations. The two major concepts - conventional, hollow-cavity (or: cylindrical-cavity) and coaxial-cavity gyrotrons - are investigated in parallel. While conventional, hollowcavity gyrotrons have a robust design and are expected to deliver around $1 \mathrm{MW}$ output power per unit [5], coaxial-cavity gyrotrons can produce up to $2 \mathrm{MW}$ (or even higher) at the cost of a more difficult design [6]. In the present study, a $236 \mathrm{GHz}$ hollow-cavity design and a $237.5 \mathrm{GHz}$ coaxial-cavity design are considered, with operating parameters as given in Table 1 [7].

In the case of the conventional-cavity concept, a thorough investigation on the highest mode eigenvalue $\chi_{\max }$ at which stable operation is still possible at $236 \mathrm{GHz}$ (assuming a diode-type start-up) was performed in order to estimate the limitations in power. This led to $\chi_{\max } \sim$ 125 [8]. The RF behaviour of the very high-order mode $\mathrm{TE}_{52,18}$ (eigenvalue $\sim 125$ ) and its competing modes was studied by multi-mode, time-dependent, self-consistent simulations using EURIDICE [9] and is shown in Fig. 2. Stable operation of the $\mathrm{TE}_{52,18}$ mode producing $\sim 1.5 \mathrm{MW}$ of power was achieved. Similar simulations for even higher-order modes verified the mode eigenvalue limit of around 125 for a $236 \mathrm{GHz}$ hollow-cavity gyrotron. The effects of an off-axis electron beam on the mode excitation and on the behaviour of neighbouring modes in the hollow-cavity design have also been studied. The simulation results suggest stable output up to a beam misalignment $D=0.30 \mathrm{~mm}(D / \lambda=0.24)$.

Although the coaxial concept is capable of higher output power, the tolerance on the misalignment of the

Table 1. Key parameters of the two gyrotron designs.

\begin{tabular}{|c|c|c|}
\hline $\begin{array}{c}\text { Parameters (ideal } \\
\text { beams) }\end{array}$ & $\begin{array}{c}\text { Coaxial } \\
\text { Concept }\end{array}$ & $\begin{array}{c}\text { Conventiona } \\
\text { l Concept }\end{array}$ \\
\hline Frequency $(\mathrm{GHz})$ & 237.5 & 236.0 \\
\hline Interaction mode & $\begin{array}{c}\mathrm{TE}_{49,29} \\
\left(\begin{array}{c}\text { Eigenvalue } \\
\approx 158)\end{array}\right.\end{array}$ & $\begin{array}{c}\mathrm{TE}_{43,15} \\
(\text { Eigenvalue } \approx \\
103)\end{array}$ \\
\hline Beam Current $(\mathrm{A})$ & 69.3 & 43 \\
\hline Beam Voltage $(\mathrm{kV})$ & 85.6 & 61 \\
\hline Magnetic Field $(\mathrm{T})$ & 9.58 & 9.175 \\
\hline Output Power $(\mathrm{kW})$ & 1900 & 920 \\
\hline $\begin{array}{c}\text { Interaction efficiency } \eta_{\text {elec }} \\
(\%)\end{array}$ & 33 & 36 \\
\hline
\end{tabular}

coaxial insert should be estimated prior to fabrication, in order to achieve robust performance. In coaxial-cavity gyrotrons, shapes and eigenvalues of modes are altered by a misalignment of the insert versus the cavity wall, a feature not present in hollow cavities. This leads to different mode eigenvalues, to changes in the coupling to the electron beam, and to increased mode conversion and thus to increased stray radiation after the end of the insert. Extensive theoretical investigations and development of numerical tools were performed in view of the study of the insert misalignment [10]. The basic idea is to express the field of a mode in a cavity with misaligned insert as a superposition of modes of a cavity with aligned insert, as shown in Fig. 3. For the coaxial design of Table 1, a maximum acceptable insert misalignment of $0.15 \mathrm{~mm}$ was estimated, since, according to calculations, such a value would lead to an additional unwanted stray radiation of $3.5 \%$, compared to about $4 \%$ stray radiation experimentally verified in a $170 \mathrm{GHz}$ coaxial cavity gyrotron.

\section{Advanced Concepts for Efficiency Enhancement}

The key to increase the gyrotron efficiency to a level significantly higher than $50 \%$ is the development of a highly efficient collector system for the recovery of the spent beam energy. In this context, a significant effort has been dedicated at KIT during the last years in order to design a reliable, robust and efficient multistage depressed collector system. There are two concepts for the efficient sorting of the spent beam electrons, which are confined by a magnetic field, on the collector stages. The first concept is based on unwinding of the spent electron beam cyclotron motion utilizing non-adiabatic transitions of magnetic fields [11], while the second one is based on ExB drift [12].

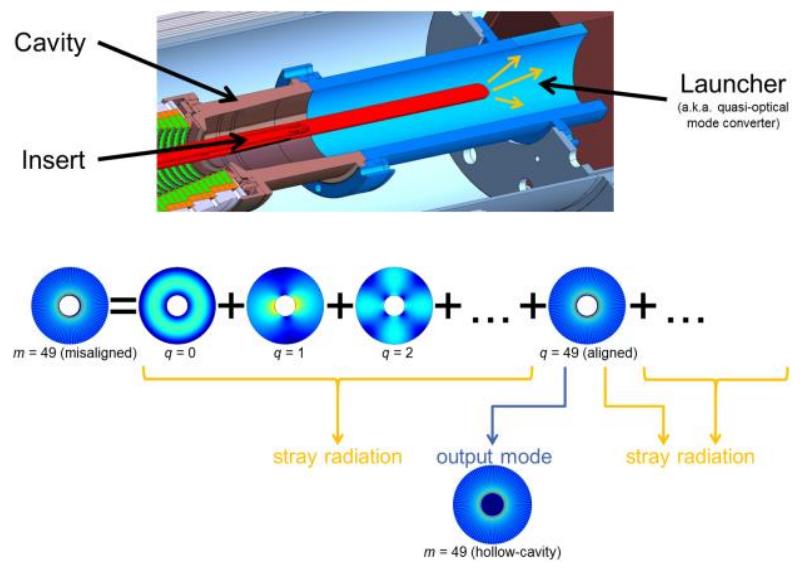

Fig. 3: Top: Cavity, insert and launcher of a coaxial gyrotron. The origin of the insert-induced stray radiation is indicated by orange arrows. Bottom: Schematic field decomposition of the misaligned-cavity mode $\mathrm{TE}_{49,29}$ into aligned-cavity modes with azimuthal indices $q=0,1,2 \ldots$ After the end of the insert, all components except for $q=49$ become stray radiation. 

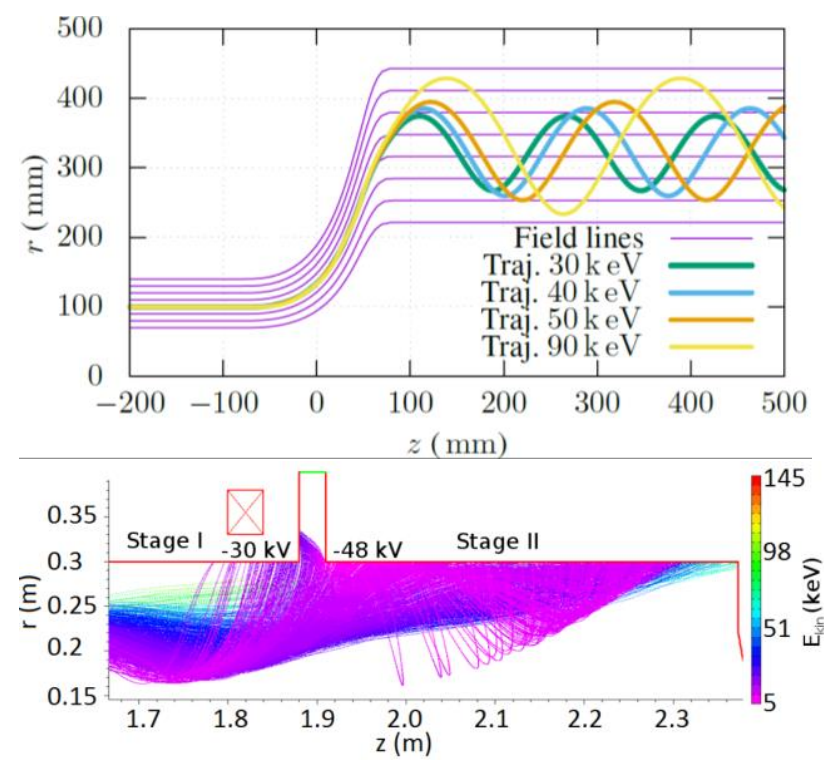

Fig. 4: Spatial modulation of electron trajectories through a non-adiabatic transition (top) and electron trajectories in the two-stage collector (bottom).

A MSDC design based on the first concept for the EU $1 \mathrm{MW} 170 \mathrm{GHz}$ gyrotron for ITER has been designed. The spent beam passes a quickly-decreasing magnetic field. After the non-adiabatic transition, two modulations appear, as shown in Fig. 4: a) the maximal reachable radii, b) the axial periodic length; both depend on the kinetic energy of electrons. Based on this modulation, we achieved $73 \%$ collector efficiency (without considering secondary electrons).

On the other hand, several design approaches have been developed based on the ExB drift concept [13, 14]. In the simplest one [15] an azimuthal electric field and the longitudinal magnetic field are used for the $\mathbf{E} \times \mathbf{B}$ drift as is schematically shown in Fig. 5. It consists of a conical helix-type slot which separates the different collector stages and induces the $\varphi$-component of the electric field. The helix slot has only one azimuthal period. The pitch of the helix defines the angle between the electric and magnetic field, and thus it is related to the strength of the $\mathbf{E} \times \mathbf{B}$ drift. Taking secondary electrons into account, PIC simulations predict an average collector efficiency of $77 \%$. Secondary electrons only reduce the efficiency by $1 \%$. There could be less than $1.5 \%$ of the total beam current returned beyond the mirror box (including secondary electrons).

\section{Conclusions}

The upgrade of the 2 MW coaxial cavity gyrotron towards longer pulses is ongoing, the design of the cooling system of the major components has been finalised, manufacturuing is ongoing. Design studies towards high frequency operation $(\sim 240 \mathrm{GHz})$ of high power gyrotrons and high efficiency operation by using multi-staged depressed collector systems were discussed.

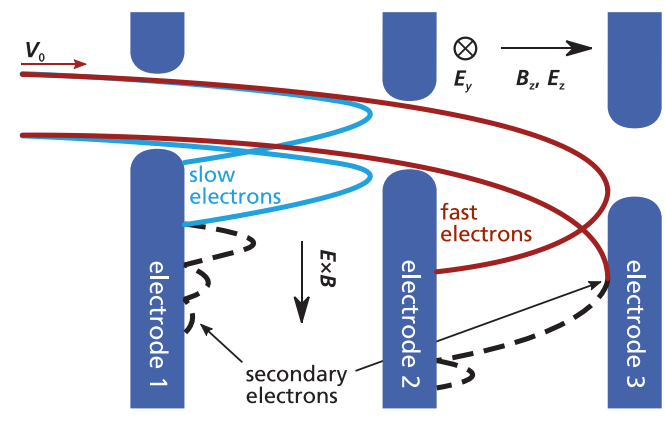

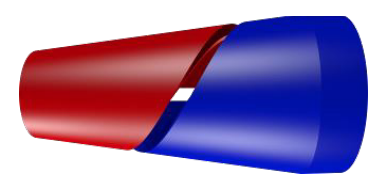

a) front side view

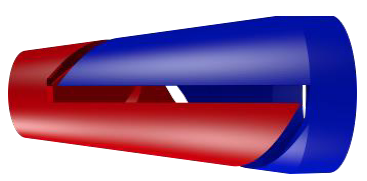

b) back side view
Fig. 5: $\mathbf{E} \times \mathbf{B}$ drift concept for the sorting of the electrons according to their initial energy (top) and two stages separated by a conical helix slot for the $\mathbf{E} \times \mathbf{B}$ drift (bottom).

\section{Acknowledgement}

This work has been partly carried out within the framework of the EUROfusion Consortium and has received funding from the EURATOM research and training programme 2014-2018 under grant agreement No 633053. The views and opinions expressed herein do not necessarily reflect those of the European Commission.

\section{References}

[1] T. Rzesnicki, et al., IEEE Transactions on Plasma Science, 38, 1141,2010

[2] I. Gr. Pagonakis et al., Physics of Plasmas 23, 23105, 2016

[3] I. Gr. Pagonakis et al., Physics of Plasmas 23, 83103, 2016

[4] S. Ruess, et al., IEEE Transaction of Electron Devices, 63, 2104, 2016

[5] P. C. Kalaria, et al., Physics of Plasmas 23, 092503, 2016

[6] J. Franck et al., 40 $0^{\text {th }}$ International Conference on Infrared Millimeter and Terahertz waves (IRMMW-THz 2015), 23-28 Aug. 2015, Hong Kong, Conference proceedings.

[7] M. Thumm, et al., Terahertz Science and Technology, 8, no. 3, pp. 85-100, September 2015.

[8] P. C. Kalaria, et al., 17th IEEE International Vacuum Electronics Conference (IVEC 2016), 19-21 April 2016, Monterey, CA, USA, Conference proceedings.

[9] K. A. Avramides, et al, EPJ Web of Conferences 32, 04016 (2012). DOI: http://dx.doi.org/10.1051/epjconf/20123204016

[10] J. Franck, Ph.D. Thesis, Karlsruher Institut für Technologie (KIT), KIT Scientific Publishing, 2017, DOI: 10.5445/KSP/1000068000.

[11] R. L. Singh, et al., United States Patent, Patent Number 5,780,970, July 1998.

[12] I. Gr. Pagonakis, et al., IEEE Trans. on Plasma Sc., 36, 469, 2008.

[13] I. Gr. Pagonakis, et al., Phys. Of Plasmas, 23, 43114, 2016.

[14] C. Wu, et al., Phys. of Plasmas, .24, pp. 43102, 2017.

[15] C. Wu, et al., 18th International Vacuum Electronics Conference (IVEC), 2017. 